\title{
Highly Effective Six-Wave Mixing in Linearly Absorbing Organic Liquids
}

\author{
I. N. Agishev and A. L. Tolstik* \\ Belarussian State University, Minsk, Belarus \\ *e-mail: tolstik@bsu.by \\ Received August 15, 2008
}

\begin{abstract}
Conditions for the realization of the highly effective transformation of a light field by dynamic holograms using a scheme of frequency-nondegenerate six-wave mixing in dyed organic liquids are determined. The mechanism of the manifestation of a nonlinear fifth-order optical susceptibility in linearly absorbing liquids is established, which is related to the nonlinearity of the thermooptical coefficient and the temperature dependence of the density and heat capacity of a solvent. The quadratic recording of dynamic holograms with a diffraction efficiency of $20 \%$ is realized in ethanol solution of copper chloride.
\end{abstract}

PACS numbers: 42.65.An, 42.65.Hw, 42.20.Pa

DOI: $10.1134 / \mathrm{S} 1063785009040221$

The expansion of the field of practical applications of the multiwave mixing methods in systems of realtime light-field transformation and optical data processing is based on the search for new media and methods that provide high values of the nonlinear optical susceptibility of the fifth and higher orders. The passage to high-order nonlinearities significantly increases the possibilities of the interference-holographic methods of processing coherent images and optical signals, including the control of the phase structure of a wave front and the frequency conversion of light fields, spatial filtration of images, and data transfer from one beam to another [1-3]. High-order nonlinearities are traditionally considered in media with multiphoton or saturable absorption [4-6], but the efficiency of six-wave mixing realized in these media does not exceed fractions of a percent, thus significantly restricting the possible practical applications of this scheme.

In this Letter, we propose for the first time to implement effective six-wave mixing using linearly absorbing liquids that are traditionally considered to be media with a purely cubic nonlinearity. Conditions for the realization of a fifth-order nonlinearity are determined and the mechanism of its manifestation is established, which is related to the nonlinearity of the thermooptical coefficient and the temperature dependences of the density and heat capacity of a solvent. These dependences are taken into account by introducing an effective second-order thermooptical coefficient, which makes possible the construction of an adequate theoretical model.

Recently, the possibility of recording quadratic dynamic holograms in clear organic liquids was pointed out [7] in the study of a six-wave interaction in ethanol. In order to explain this phenomenon, it was suggested to take into consideration the temperature dependence of thermooptical characteristics, which could account for the saturation of the thermal nonlinearity by analogy with the behavior of resonance media possessing saturable absorption. However, with this phenomenological approach to saturation, it is difficult to quantitatively determine the parameter characterizing this nonlinearity.

In order to describe the process of diffraction on thermal diffraction gratings under the conditions of linear absorption, we propose to expand the refractive index as a function of the temperature into Taylor's series in derivatives of various orders and to take into account the temperature dependence of the density and heat capacity of an organic liquid. In this case, a temperature-induced change in the refractive index can be expressed as follows:

$$
\Delta n=(\partial n / \partial T) \Delta T+\frac{1}{2}\left(\partial^{2} n / \partial T^{2}\right)_{\mathrm{eff}}(\Delta T)^{2}+\ldots,
$$

where $\partial n / \partial T$ and $\partial^{2} n / \partial T^{2}$ are thermooptical coefficients of the first and second order, respectively; $\Delta T=k I \tau / C_{\rho}$ is a change in the temperature; $k$ is the absorption coefficient; $I$ is the intensity; $\tau$ is the pulse duration; and $C_{\rho}$ is the heat capacity per unit volume. In Eq. (1), we introduce the effective second-order thermooptical coefficient that takes into account the temperature dependence of the heat capacity and density, which is defined as follows:

$$
\left(\partial^{2} n / \partial T^{2}\right)_{\mathrm{eff}}=\partial^{2} n / \partial T^{2}-\left(\partial C_{\rho} / \partial T\right)(\partial n / \partial T) C_{\rho}^{-1} .
$$

The first term in the right-hand side of Eq. (1) describes the cubic nonlinearity (a change in the refractive index is proportional to the intensity), while the second term takes into account the quadratic depen- 


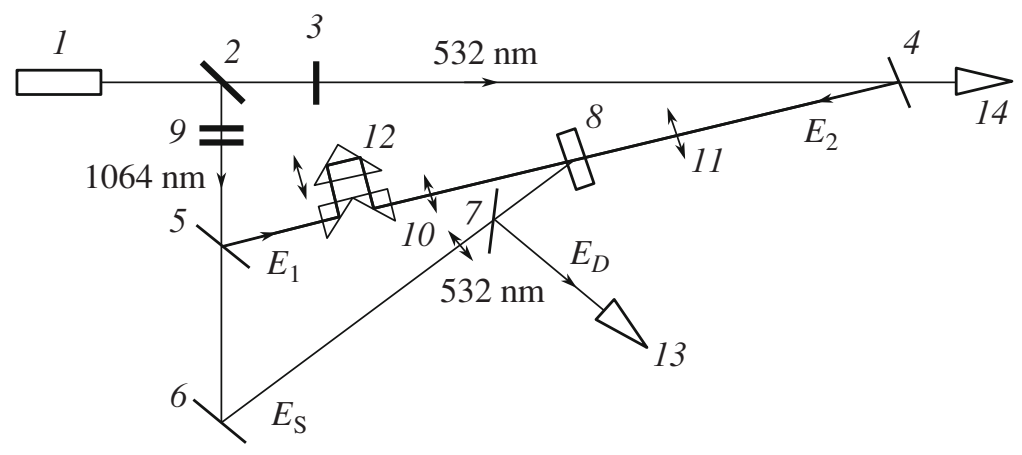

Fig. 1. Schematic diagram of the experimental setup for studying six-wave mixing (see text for explanations).

dence of the refractive index on the intensity and is uniquely related to the fifth-order nonlinearity and sixwave mixing as

$$
\chi^{(5)}=\frac{c^{2} n_{0}^{3} k^{2} \tau^{2}}{256 \pi^{3} C_{\rho}^{2}}\left(\partial^{2} n / \partial T^{2}\right)_{\mathrm{eff}},
$$

where $n_{0}$ is the initial refractive index of the linearly absorbing organic liquid.

Using Eqs. (1) and (2), it is possible to calculate the efficiency of the six-wave mixing. Figure 1 shows the typical scheme of this interaction. A dynamic hologram was recorded by waves $E_{1}$ and $E_{\mathrm{S}}$ at the fundamental laser frequency $\omega$, while the readout was performed by wave $E_{2}$ at the double frequency $2 \omega$. The Bragg diffraction condition for the six-wave mixing is satisfied for counterpropagating $E_{1}$ (reference) and $E_{2}$ (readout) waves in the scheme of wave front reversal with frequency doubling $[8,9]$. In this case, the diffracted wave $E_{D}$ is determined by the nonlinear polarization $P=$ $\chi^{(5)}\left(E_{1} E_{\mathrm{S}}^{*}\right)^{2} \mathrm{E}_{2}$, which corresponds to the quadratic recording of dynamic holograms.

Theoretical description of the frequency-nondegenerate six-wave mixing is given in the approximation of slowly varying amplitudes, which yields the following system of truncated wave equations for the interacting light fields [5]:

$$
\begin{gathered}
\frac{\partial E_{1, \mathrm{~s}}}{\partial r}=i \frac{2 \pi \omega}{c n_{0}}\left[E_{1, \mathrm{~s}} \chi_{0}(\omega)+E_{\mathrm{S}, 1} \chi_{ \pm 1}(\omega)\right], \\
\frac{\partial E_{2, D}}{\partial r}=-i \frac{4 \pi \omega}{c n_{0}}\left[E_{2, D} \chi_{0}(2 \omega)+E_{D, 2} \chi_{\mp 2}(2 \omega)\right],
\end{gathered}
$$

where $\chi_{m}$ are the components of expansion of the nonlinear susceptibility into the Fourier series with respect to spatial harmonics of the dynamic grating $\mathbf{K}=\mathbf{k}_{1}-\mathbf{k}_{\mathbf{s}}$. In the approximation of small diffraction efficiency, Eq. (4) reduces to the following relation:

$$
\frac{\partial E_{D}}{\partial r}=-i \frac{4 \pi \omega}{c n_{0}} \chi^{(5)}\left(E_{1} E_{\mathrm{S}}^{*}\right)^{2} E_{2},
$$

This equation yields the following expression for the diffraction efficiency:

$$
\xi=\frac{I_{D}}{I_{2}}=\left(\frac{4 \pi \omega L}{c n_{0}}\right)^{2}\left|\chi^{(5)}\right|^{2}\left(I_{1} I_{\mathrm{S}}\right)^{2}\left(\frac{8 \pi}{c n_{0}}\right)^{4}
$$

where $L$ is the thickness of the nonlinear layer.

Figure 1 presents a schematic diagram of the experimental setup. The radiation source was a pulsed yttrium aluminum garnet laser 1 operating in the active $Q$-switching regime at a pulse duration of $\tau=20 \mathrm{~ns}$. The dynamic hologram was recorded at the fundamental laser frequency $(\lambda=1064 \mathrm{~nm})$ and read at the second harmonic $(\lambda=532 \mathrm{~nm})$. The spectral separation of beams was provided by beam divider 2 . Mirrors 5 and 6 and lenses 10 formed the reference $\left(E_{1}\right)$ and signal $\left(E_{\mathrm{S}}\right)$ beams with a diameter of $650 \mu \mathrm{m}$, which were used to record the dynamic grating in nonlinear medium 8 . The angle between these beams $(\sim 100 \mathrm{mrad})$ ensured the almost complete wave overlap in a 1-mm-thick cell. The time of the arrival of recording laser radiation pulses was synchronized using delay line 12. Mirror 4 formed readout wave $E_{2}$ propagating in the direction opposite to that of the recording waves; lens 11 created a neck with a diameter of $350 \mu \mathrm{m}$. The wave intensities were controlled using filters 3 and 9. The diffraction efficiency was measured using the system of detectors 13 and 14. In our experiments, the nonlinear medium was a solution of copper chloride in ethanol, which is characterized by a high thermal nonlinearity. Another advantage of this medium is a relatively high absorption in the near-IR spectral range as compared to that in the visible range (the absorption coefficients at 1064 and $532 \mathrm{~nm}$ differ by more than an order of magnitude).

Figure 2 shows plots of the diffraction efficiency $\xi=$ $I_{D} / I_{2}$ of the quadratic dynamic hologram versus wave intensity and optical thickness of the medium. As can be seen, the diffraction efficiency in the region of its small values is proportional, in accordance with formula (6), to the fourth power of the recording wave intensity and to the square of the optical thickness (the corresponding parabolas are depicted by solid lines in Fig. 2). The diffraction efficiency begins to saturate on 

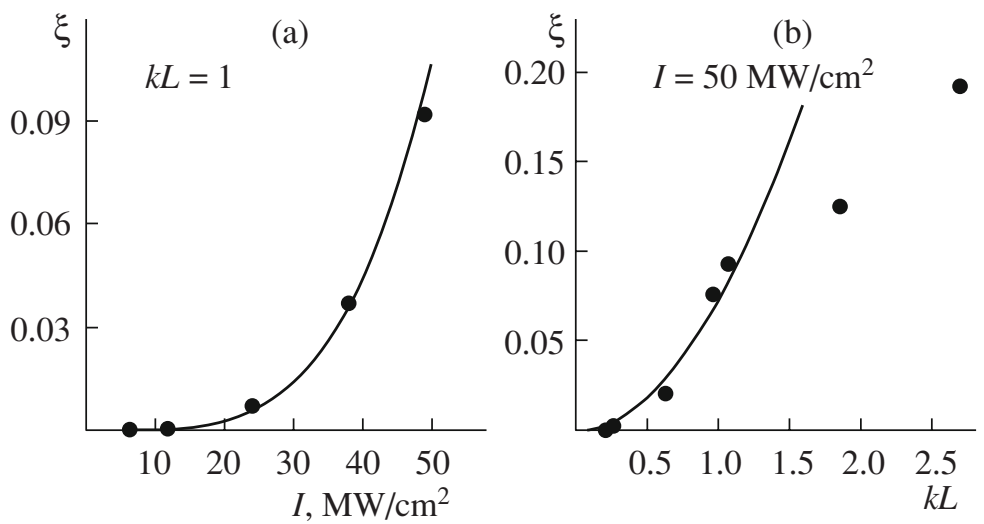

Fig. 2. Plots of the diffraction efficiency $\xi$ of the quadratic dynamic hologram versus (a) wave intensity $I_{1}=I_{\mathrm{S}}=I$ and (b) optical thickness $k L$ of the medium. Points present the experimental data and solid lines show the fitting model parabolas.

a level above $10 \%$. The maximum $\xi$ value achieved in these experiments was about $20 \%$, which is several orders of magnitude higher than the values reported previously for the frequency-nondegenerate six-wave mixing based on the two-photon absorption in polymers [4] and the saturable absorption in organic dye solutions [5] and clear organic liquids [7].

The adequacy of the proposed approach to description of the frequency-nondegenerate six-wave mixing in linearly absorbing liquids was confirmed by comparing the experimental data to the results of theoretical calculations. In particular, by substituting the results of experiments into formulas (2) and (6), the effective second-order thermooptical coefficient was evaluated at $\left(\partial^{2} n / \partial T^{2}\right)_{\text {eff }} \approx 10^{-6} \mathrm{~K}^{-2}$. According to the LorentzLorenz formula, the second-order thermooptical coefficient is $\partial^{2} n / \partial T^{2} \approx-10^{-7} \mathrm{~K}^{-2}$, while the tabulated data [10] yield the heat capacity per unit volume of ethanol $C_{\rho}=1.92 \mathrm{~J} /\left(\mathrm{K} \mathrm{cm}^{3}\right)$, the first derivative $\partial C_{\rho} / \partial T \approx 5 \times$ $10^{-3} \mathrm{~J} /\left(\mathrm{K}^{2} \mathrm{~cm}^{3}\right)$, and the corresponding first-order thermooptical coefficient $\partial n / \partial T=-4 \times 10^{-4} \mathrm{~K}^{-1}$. Then, the published data yield an estimate of the effective second-order thermooptical coefficient $\left(\partial^{2} n / \partial T^{2}\right)_{\text {eff }}=$ $\partial^{2} n / \partial T^{2}-\left(\partial C_{\rho} / \partial T\right)(\partial n / \partial T) C_{\rho}^{-1}$, which is close to our experimental value.

In conclusion, we demonstrated good prospects for the use of linearly absorbing dyed organic liquids as media for the realization of highly effective six-wave mixing on fifth-order nonlinearities. It is established that the main contribution to this interaction is related to the thermal nonlinearity caused by the temperature dependences of the density and heat capacity of a solvent.

\section{REFERENCES}

1. K. D. Merkel, Z. Cole, and W. R. Babbiti, J. Luminesc. 86, 375 (2000).

2. V. Astinov, K. J. Kubarych, C. J. Milne, and R. J. D. Miller, Optics Lett. 25, 853 (2000).

3. O. Ormachea, O. G. Romanov, A. L. Tolstik, et al., Optics Express 14, 8298 (2006).

4. F. Charra and J.-M. Nunzi, J. Opt. Soc. Am. B 8, 570 (1991).

5. A. S. Rubanov, A. L. Tolstik, S. M. Karpuk, and O. Ormachea, Optics Commun. 181, 183 (2000).

6. Y.-F. Chen, K. Beckwitt, F. W. Wise, B. G. Aitken, J. S. Sanghera, and I. D. Aggarwal, J. Opt. Soc. Am. B 23, 347 (2006).

7. O. Ormachea and A. L. Tolstik, Izv. Ross. Akad. Nauk, Ser. Fiz. 71, 131 (2007) [Bull. Russ. Acad. Sci., Phys. 71, 126 (2007)].

8. V. V. Ivakhnik and V. I. Nikonov, Opt. Spektrosk. 75, 385 (1993) [Opt. Spectrosc. 75, 227 (1993)].

9. S. M. Karpuk, A. S. Rubanov, A. L. Tolstik, and A. V. Chalei, Pis'ma Zh. Tekh. Fiz. 20 (12), 4 (1994) [Tech. Phys. Lett. 20, 475 (1994)].

10. V. I. Stabnikov, I. M. Roiter, and T. B. Protsyuk, Ethyl Alcohol (Pishchevaya Prom-st, Moscow, 1976) [in Russian].

Translated by P. Pozdeev 